\title{
Investigation of bubble behaviours in wet central heating systems
}

\author{
Ali Shefik ${ }^{1, a}$ and Yunting $\mathrm{Ge}^{1}$ \\ ${ }^{1}$ Brunel University, Department of Mechanical Engineering, UB8 3PH, United Kingdom
}

\begin{abstract}
A series of experimental measurements has been conducted in order to investigate the bubble behaviours through the horizontal pipe line of the domestic wet central heating systems. Obtained results exposed the effect of 90 degree bend, buoyancy forces on bubbly two phase flow patterns and effect of velocity on void fractions and bubble diameters. Distance chosen for the first sight glass (HSG0) was sufficient enough to note the effect of 90 degree bend on void fraction patterns. Due to the effect of 90 degree bend, position of the peak void fractions across the pipe section lowers, with an increase in bulk fluid velocity. Bubbles tend to flow for longer distance at the bottom of the pipe section. Buoyancy force effect is demonstrated with figures for highest bulk fluid velocity at three different positions. Analysis of four different flow rates at two different saturation ratios show reduction for average bubble diameters and void fractions when bulk fluid velocity increases. An attempt to predict bubble dissolution rates across the horizontal pipeline of the system is made, however results show some uncertainties.
\end{abstract}

\section{Introduction}

Bubble formation in wet central heating systems is due to the supersaturated conditions which occur on the primary heat exchanger wall of the boiler. Dissolved gases in the flowing liquid are released as bubbles when they encounter high temperatures. Subsequently, these bubbles tend to escape to the higher parts of the system (low pressure) and cause cold spots, and maintenance or noise problems. It is an increasing trend in building services to install devices through the pipeline of the systems for removing dissolved gases from the working fluid to prevent unwanted events, and therefore save time and energy. Little data exists in the literature for two-phase flow characteristics, particularly for central heating systems, to assist companies to improve such devices. In this regard, investigation of bubble behaviours in central heating systems becomes important to help enhance the design of deaerators.

For this purpose, the test rig shown in figure 4 was established at Brunel University to represent an ordinary wet domestic central heating system and series of experiments are conducted in order to obtain a better understanding of two-phase flow phenomenon in such systems. Current project is taken over from an earlier researcher who gathered useful data on typical bubble sizes, volumetric void fractions, bubble distributions and nucleation and dissolution rates [1, 2, 3]. Our aim in the presently on-going project is to extend the information on bubble distribution across the pipe section and bubble size differences through both horizontal and vertical pipes of the system and adapt the general bubble dissolving models proposed by Lezhnin et al. [4] and Shedd [5] to predict the bubble size alterations throughout the pipeline. In the following phase of the project, computational fluid dynamics (CFD) software Fluent will be used to simulate mass transfer effect from fluid to bubbles or vice versa.

\section{Approach}

\subsection{Theory}

\subsubsection{Solubility of gases in liquids}

Henry's law (Equation 1) states that the amount of dissolved gas concentration in a liquid is directly proportional with the partial pressure of the gases in the liquid [6]. Temperature dependent Henry's constant $\left(X^{T}\right)$ can be read from the solubility tables.

$$
C_{\text {gas }}=p_{\mathrm{g}} X^{\mathrm{T}}
$$

Actual dissolved gas concentration $\left(C_{\mathrm{act}}\right)$ in liquid can be calculated through the use of Equation 1 if the partial gas pressure and temperature of the liquid is known (Equation 2). In our case, these parameters were obtained by the total gas measurement device (TGM).

$$
C_{\mathrm{act}}=\left(p_{\mathrm{TGM}}-p_{\mathrm{vap}}\right) X_{\mathrm{TGM}}^{\mathrm{T}}
$$

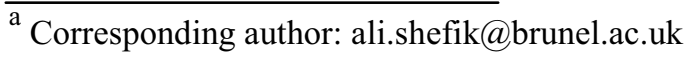


Maximum amount of gas which can be dissolved into liquid $\left(C_{\mathrm{sat}}\right)$ at specified liquid temperature and pressure $\left(T_{\mathrm{L}}\right.$ and $\left.P_{\mathrm{L}}\right)$ can be calculated using Equation 3 .

$$
C_{\text {sat }}=\left(P_{\mathrm{L}}-p_{\text {vap }}\right) X_{\mathrm{L}}^{\mathrm{T}}
$$

Saturation ratio $(\alpha)$ is defined as the ratio of actual dissolved gas concentration in the system to maximum dissolved gas concentration at specified temperature and pressure [7]. In the following parts of this article, maximum dissolved gas concentration is calculated according to the readings obtained from the temperature and pressure sensors placed before the HSG0 $\left(T_{1}\right.$ and $\left.P_{1}\right)$.

$$
\alpha=C_{\text {act }} / C_{\text {sat }}
$$

Figure 1 visualizes the change of dissolved air concentration in water with respect to temperature at 2.7 bar. If we start from saturated state (Position A) and start to heat the liquid at constant pressure, the solution will eventually come the point B which is supersaturated (oversaturated) solution. At this point, solution has a tendency to produce bubbles and reduce the amount of dissolved air in the liquid to reach equilibrium state (saturated state). If one continues to force solution for degassing (reducing temperature or increasing pressure) the solution will become undersaturated.

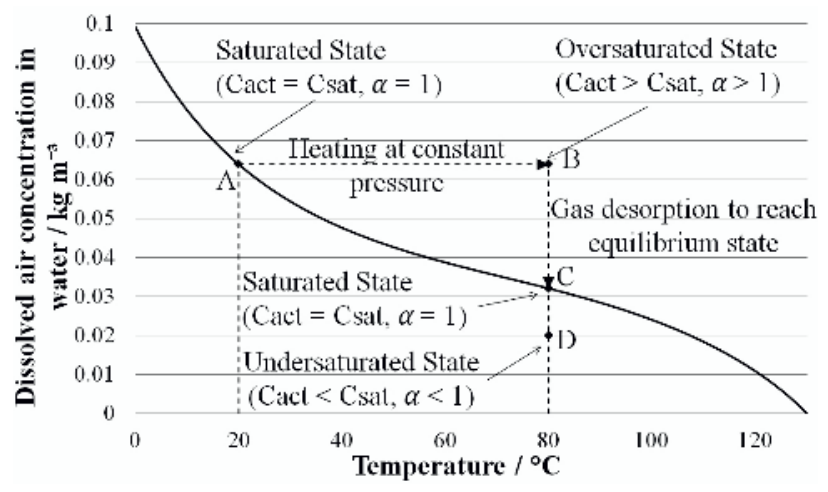

Figure 1. Solubility of air in water at 2.7 bar

\subsubsection{Bubble dissolution model}

Mass flux on bubble boundary is driven by the concentration difference between bubble boundary and ambient fluid where $\mathrm{k}$ indicates the mass transfer coefficient [8].

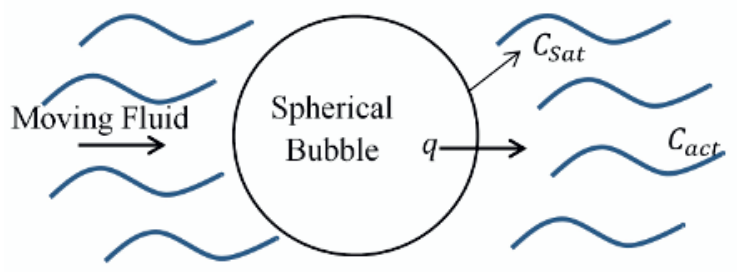

Figure 2. Mass transfer from bubble to its ambient fluid

$$
q=k\left(C_{\mathrm{sat}}-C_{\mathrm{act}}\right)
$$

Sherwood number $\left(S h=k d_{\mathrm{b}} / D_{A B}\right)$ is used to find the mass transfer coefficient $(k)$ for convective mass transfer. Different empirical Sherwood number calculations $[4,5]$ found in literature will be compared in this study to identify the most successful one. A, B and C are constants for empirical correlation [8].

$$
S h=\mathrm{A}\left(d_{b} / L\right) R e^{\mathrm{B}} S c^{\mathrm{C}}
$$

Different empirical formulas result with different mass transfer coefficient $(k)$ calculations. Figure 3 was plotted using the particular parameters for the case studied in this article $(20 \mathrm{~mm}$ inlet pipe diameter and 80 $\left.{ }^{\circ} \mathrm{C}\right)$. It can be noticed that the main factor affecting the coefficient calculation is Reynolds constant B. This is mainly due to the fact that empirical equations found in literature for calculating the Sherwood number are very sensitive to Reynolds number.

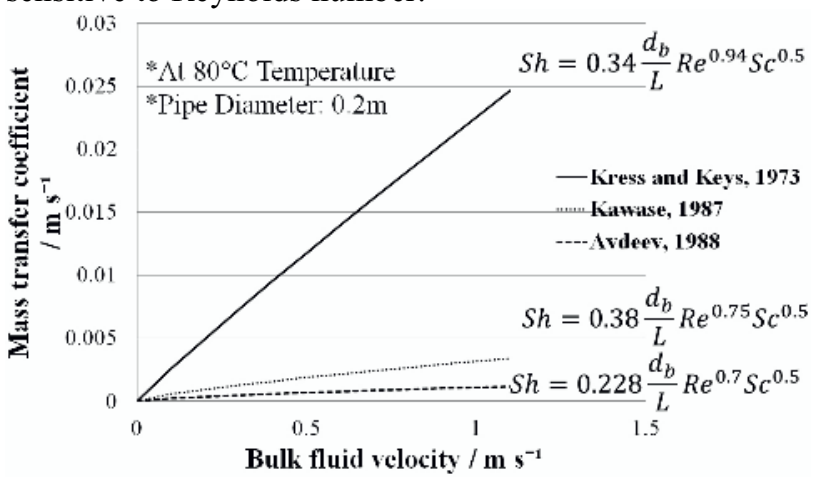

Figure 3. Comparison of the empirical Sherwood formulas

Moreover, binary diffusion constant for gas species A in liquid $\mathrm{B}\left(\mathrm{m}^{2} \mathrm{~s}^{-1}\right)$, which is used in the definition of Sherwood Number, is estimated with empirical formula purposed by C.R. Wilke and P. [9].

\subsection{Experimental set-up and system conditions}

\subsubsection{Experimental set-up}

The test rig in figure 4 shows all the equipment that was used in project experiments. Vaillaint ecoTec pro 24 condensing boiler provides necessary energy to heat up the flowing liquid. Conditions of the system were monitored by five K-type thermocouples, four pressure transducers and an electromagnetic flow meter. All of the sensors were calibrated before the experiments, with appropriate devices to make sure that acquired date is reliable. The signals from the measurement equipment were collected with data acquisition device (National Instruments cDAQ-9172) and were monitored with the LapView software on PC monitor.

The rig consists of two closed loops which are connected at the buffer vessel. The primary loop is the main part of the test rig, where filming of the fluid flow takes place through sight glasses by high speed camera. Figure 6 shows a more detailed setup of the camera and lighting positions. The secondary loop of the system is used to measure the partial pressure of dissolved gases in working fluid and helps us to calculate actual dissolved gas concentration $\left(C_{\text {act }}\right)$ in the flow by Henry`s law (Equation 2). Gas partial pressure measurements are done via a device called TGM (Total gas measurements) which 


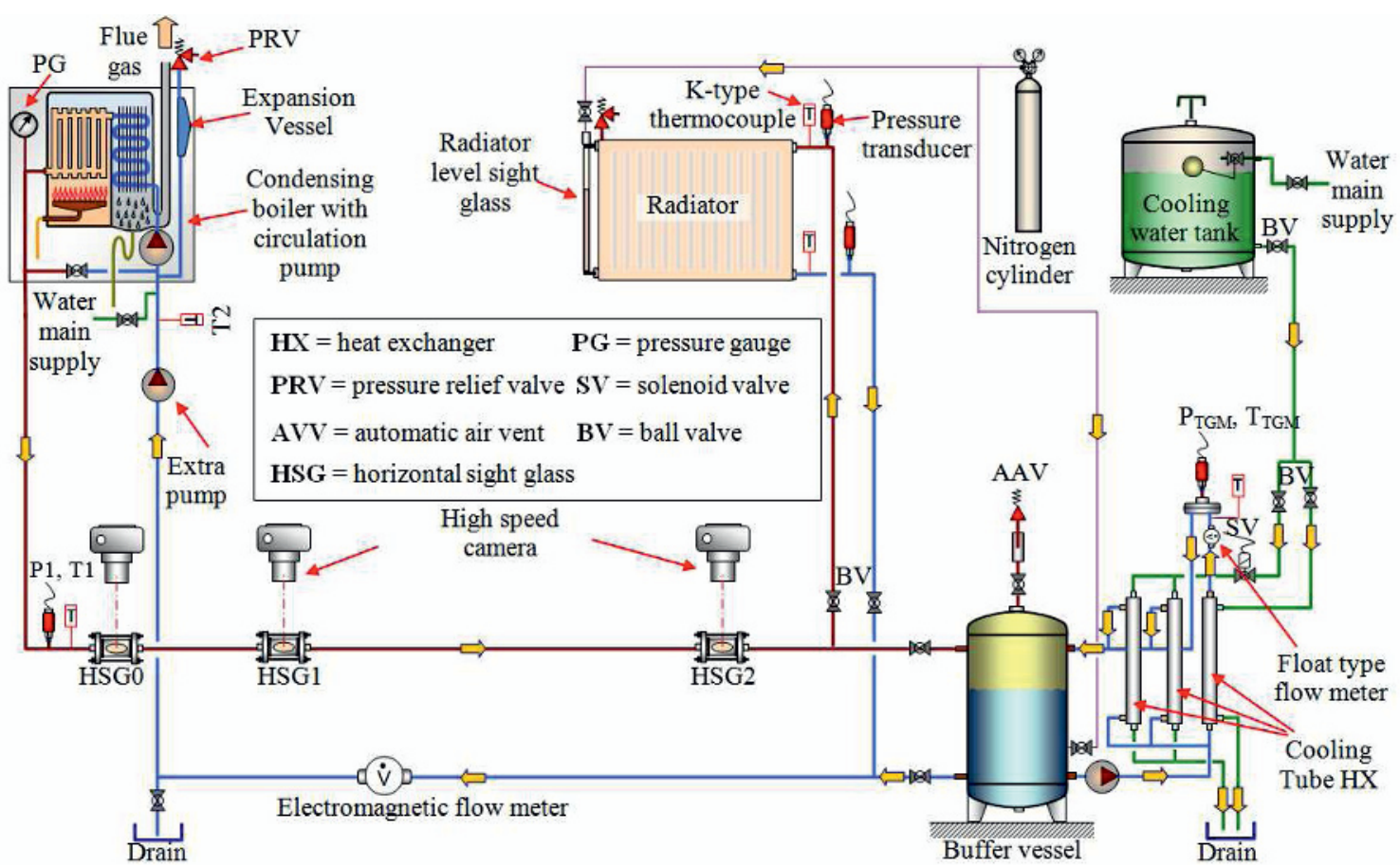

Figure 4. Schematic diagram of the test rig.

uses semi-permeable silicone membrane. Semipermeable membrane allows gas particles to pass through and reach the pressure transducer while it blocks liquid state to reach pressure sensor. TGM requires fluid temperatures to be between $20^{\circ} \mathrm{C}$ and $45^{\circ} \mathrm{C}$. Therefore, system fluid is cooled down with tap water cooling tube heat exchanger before it reaches the TGM. The secondary loop also enables us to control the boiler exit temperature $\left(T_{1}\right)$ with the solenoid valve which turns on and off the cooling water.

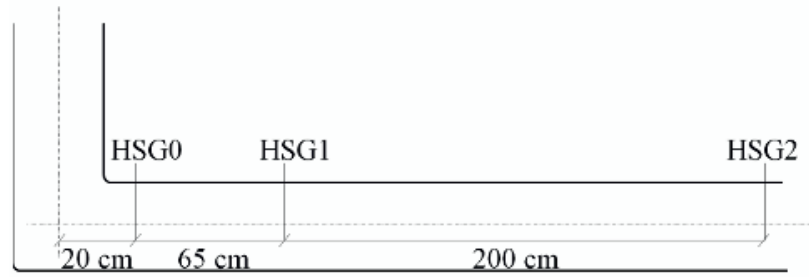

Figure 5. Three (HSG0, HSG1 and HSG2) different measurement positions at horizontal pipe (20 $\mathrm{mm}$ diameter)

The sight glasses were installed on the first horizontal pipeline of the system after the boiler exit. Distances between the sight glasses and the bend (Figure 5) will produce information on bubble distribution across the pipe after the bend; and distances of the sight glasses relative to each other are expected to produce bubble size alterations between the sight glasses, according to the saturation level $(\alpha)$ of the flow. If the fluid flow is under saturated $(<1)$ the bubble sizes are expected to get smaller, whereas if the flow is oversaturated $(>1)$, an increase should be observed through the pipeline because of mass transfer occurring on the boundary of bubbles.

Firstly, the images at HSG0 were captured for each saturation ratio and flow rate. After the measurements were done at HSG0, the position of the sight glass and lighting setup were changed to the next position (HGS1) and filming of the flow was continued. Lastly, same procedure was repeated for HSG2 to complete the experiments.

\subsubsection{System conditions}

The fluid flow filmed for 2 different saturation ratios at 4 different flow rates. Flow rate was changed with the help of ball valves and an extra pump. Saturation level was controlled by injecting/removing nitrogen and changing system pressure by adding/removing water to/from the system. Other system conditions during the measurements like heat load $(Q)$, boiler exit temperature $\left(T_{1}\right)$, pressure $\left(P_{1}\right)$ etc. were kept constant to enable procurement of comparable data.

Maximum boiler heat load and target boiler exit temperature were set as $10 \mathrm{~kW}$ and $85{ }^{\circ} \mathrm{C}$ respectively from controls on the boiler. Controlling of the fluid temperature before HSG0 $\left(T_{1}\right)$ is performed by the solenoid valve (SV in figure 4) which turns cooling water on and off to two of the tube heat exchangers according to the temperature reading from the first thermocouple of the system $\left(T_{1}\right)$. Threshold level for solenoid valve to turn on/off was set as $80{ }^{\circ} \mathrm{C}$ from the interface of LabVIEW software which visualizes the measured signals from the thermocouples, pressure sensors and flow meter. We did not allow boiler to turn off itself after reaching target boiler exit temperature $\left(85^{\circ} \mathrm{C}\right)$ by setting the threshold level $80{ }^{\circ} \mathrm{C}$ at point $T_{1}$, hence enabling us to have control over system temperature. Having control on cooling water allows us to maintain fluid temperature before HSG0 at $80^{\circ} \mathrm{C}$ and work at constant heating load.

The day before the experiments, an injection of nitrogen gas through buffer vessel and radiator was applied to increase gas concentration in the system. It takes a long time for gases to dissolve into water, so the cold cycle through whole night helps us to reach 
sufficient dissolved nitrogen amounts next morning. When the boiler is turned on next morning, high saturation ratios are observed and they reduce by the time because bubbles which form on the boiler primary heat exchanger wall tend to escape to low pressure parts of the system. They either accumulate on the upper part of the radiator or escape outside through the AVV (Automatic air vent) which is on the top of buffer vessel (Figure 4). The measurements were done when the system conditions matched with desired conditions compiled in Table 1. Measurements were done for one flow rate each day, in order to follow the same procedure for all the measurements, since changing the flow rate while system is running has significant effects on system conditions. Heating load $(Q)$ was calculated with Equation 5 to check if it shows consistency with the heating load that was set on boiler display $(10 \mathrm{~kW})$.

$$
Q=\dot{m} c_{\mathrm{p}} \Delta T
$$

Table 1. System conditions during the measurements

\begin{tabular}{|c|c|c|c|c|c|c|}
\hline $\begin{array}{c}\boldsymbol{U} \\
\left(\mathbf{m s}^{-1}\right)\end{array}$ & $\begin{array}{c}\boldsymbol{R e} \\
(-)\end{array}$ & $\begin{array}{c}\boldsymbol{T}_{\mathbf{1}} \\
\left({ }^{\circ} \mathbf{C}\right)\end{array}$ & $\begin{array}{c}\boldsymbol{T}_{\mathbf{2}} \\
\left({ }^{\circ} \mathbf{C}\right)\end{array}$ & $\begin{array}{c}\boldsymbol{P}_{\mathbf{1}} \\
(\mathbf{b a r})\end{array}$ & $\begin{array}{c}\boldsymbol{Q} \\
(\mathbf{k W})\end{array}$ & $\begin{array}{c}\boldsymbol{S R} \\
( \pm \mathbf{0 . 1})\end{array}$ \\
\hline 0.531 & 21619 & 80 & 63 & 2.7 & 11.59 & $\begin{array}{c}0.9 \\
0.75\end{array}$ \\
\hline 0.663 & 28497 & 80 & 67 & 2.7 & 11.07 & $\begin{array}{c}0.9 \\
0.75\end{array}$ \\
\hline 0.884 & 37996 & 80 & 70 & 2.7 & 11.35 & $\begin{array}{c}0.9 \\
0.75\end{array}$ \\
\hline 1.105 & 47495 & 80 & 72 & 2.7 & 11.35 & $\begin{array}{c}0.9 \\
0.75\end{array}$ \\
\hline
\end{tabular}

\subsection{Camera measurements and image analyses}

\subsubsection{Camera setup and settings}

Camera and lighting positions given in figure 6 were used as a reference to adjust camera and lights in order to have identical standings during each measurement. The detailed camera setup in figure 6 also includes sample fluid volumes which are filmed for 10 focal planes. First focal plane started just underneath the sight glass and moved down with $2 \mathrm{~mm}$ intervals each time by the help of the camera slider. These planes are used to obtain bubble distributions across the pipe section.

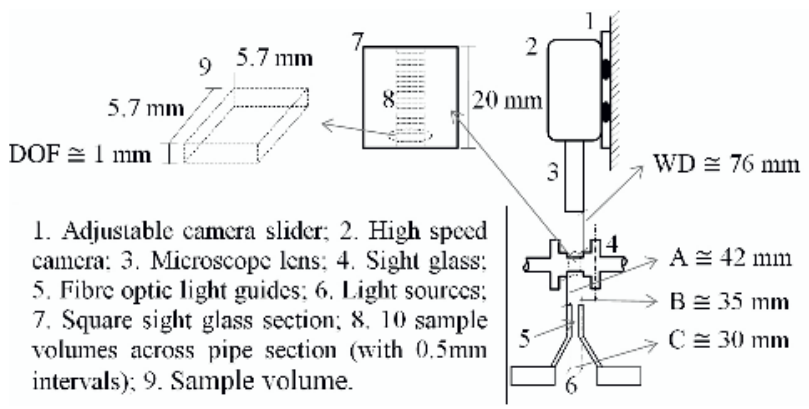

Figure 6. Camera setup and sample volume with $1 \mathrm{~mm} \mathrm{DOF}$

The camera and zoom settings are given in table 2 were used during the experiments and were chosen according to the results of several experiments. The frame size and image quality were taken into consideration while choosing the camera and lens settings.

Table 2. Camera and zoom settings

\begin{tabular}{|c|c|}
\hline Camera used & Phantom V5.1 High Speed Camera \\
\hline Zoom & Leica Monozoom 7 \\
\hline Objective used & $3 \mathrm{x}$ \\
\hline Zoom level & $512 \times 512$ \\
\hline Resolution & $40 \mathrm{FPS}$ \\
\hline Sample rate & $2 \mu \mathrm{s}$ \\
\hline Exposure time & $1 \mu \mathrm{s}$ \\
\hline EDR exposure & $1 \mathrm{p}$ \\
\hline Post trigger & \\
\hline
\end{tabular}

\subsubsection{Depth of field determination}

Depth of field (DOF) is the thickness of the volume, where acceptable sharp objects can be captured by the camera with its specified zoom level and settings. The objects within volume appear clear and sharp in the image whereas objects that are out of this volume appear blurry.

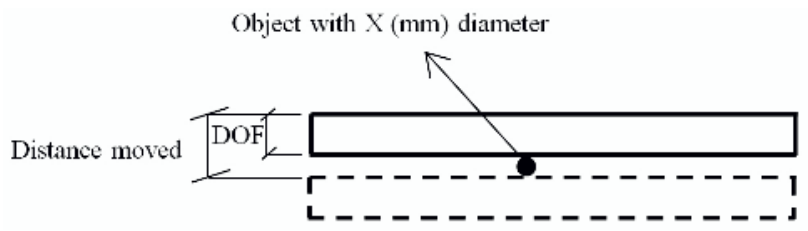

Figure 7. Depth of field representation

Figure 7 shows a representation of how DOF can be calculated and determined. The rectangle represents the depth of the volume which camera can capture images bright and clear. It moves up or down with respect to camera position and if we assume that the diameter of the object is too small, DOF will be equal to the distance moved.

$$
\text { Distance moved }=\text { DOF }+ \text { Object diameter }
$$

Determining the DOF for the measurements in this paper was done with tests under stagnant bubbles where they were just underneath the glass. The camera and the sight glass position were set as shown in Figure 6 and the boiler was turned off suddenly during the oversaturated conditions to obtain stagnant bubbles just underneath the glass. Frames were taken with different working distances (WD) from high to low and the images were analysed according to brightness and sharpness of the stagnant bubbles. The DOF was determined approximately as $1 \mathrm{~mm}$ for specific camera and zoom settings with the data and assumptions applied in Equation 8. 


\subsubsection{Image analyses}

In the image analysing process, 1000 frames (100 per focal plane) for each sight glass at each condition were analysed. Set of images (100 frames) for every focal plane were saved as a .cine file and converted to 100 .tiff files with the software called Cine Viewer. Then the frames obtained from the experiments were analysed by image processing software Image-Pro. Calibration of the image processing software is needed to gather meaningful data. Small metal balls (1 $\mathrm{mm}$ diameter) were chosen to identify the reference length for image processing software.

An automated route was recorded in order to reduce analysis period and gather the diameters of the bubbles that are in frames. During the automated route, several enhancement methods such as background corrections, flatten, sharpen and sobel filter, were used to improve the processing stage.

Background correction method was used to remove the spots on the glass since exposing the bubbles which are the main interest of our research. Figure 8 illustrates an example of background correction used in the analyses.
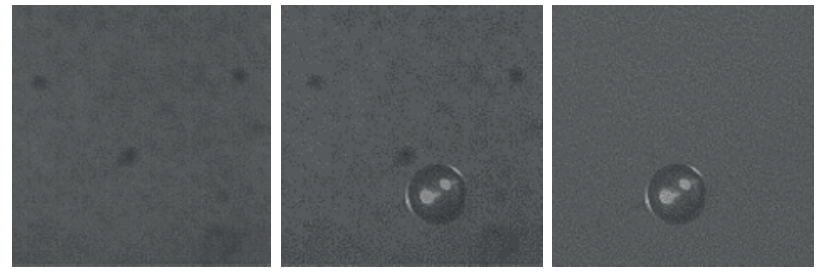

Figure 8. Effect of background correction

Flatten and sharpen filters were used to even out the background intensity variations to improve the effect of sobel filer, which extracts and enhances the edges of every pixel with respect to its neighbouring pixel. Sobel filter was used to identify the difference between the bubbles that are in focus and out of focus. Bubbles which are in focus have high intensity gradients with respect to its background, whereas bubbles that are out of focus do not. Figure 9 demonstrates the effect of flatten, sharpen and sobel filters [10].
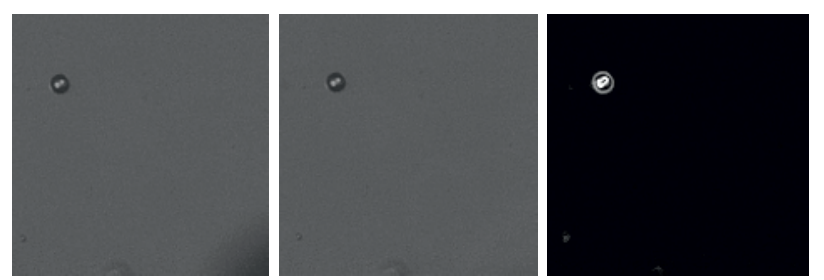

Figure 9. Effect of flatten, sharpen and sobel filter

Finally, figure 10 illustrates a typical image after automated route has been used on random set of experiment data.

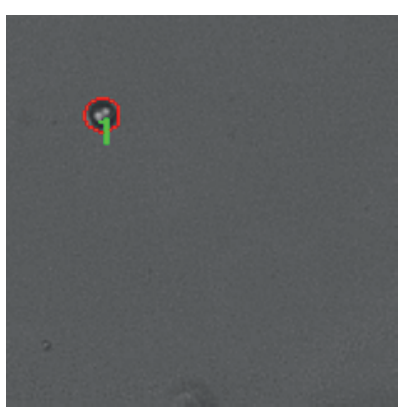

Figure 10. Typical image after the image analyses

\section{Results}

Bubble distributions across pipe section are shown in the following $\log$ of void fraction versus focal plane figure 11-13. Void fraction is described as the ratio of bubble volume to analysed sample volume (9).

$$
\text { Void Fraction }=\mathrm{V}_{\text {Tbub }} / \mathrm{V}_{\mathrm{Tsam}}
$$

Focal plane depth is defined as a distance from the top of the pipe; where $0 \mathrm{~mm}$ focal plane depth refers to the first plane, just underneath the glass, and $18 \mathrm{~mm}$ is the last plane, $2 \mathrm{~mm}$ above from the glass at the bottom of the pipe.

Arithmetic average is used to calculate average bubble diameter values presented at the y-axes in figure 14 and 15.

\subsection{Bubble distribution after 90 degree bend}

\subsubsection{Bend effect at HSGO (SRO.75 and SR0.9)}

Figure 11 and 12 visualize the change of the void fraction with respect to the bulk velocity change at HSG0. Identical findings can be reached from both results for saturation ratio 0.75 and 0.9 . The peak void fraction trend from the top of the pipe to bottom of the pipe is very similar. Peak void fraction positions at HSG0 gets closer at the bottom of the pipe with an increase in bulk fluid velocity. This shows that with the effect of high fluid velocities and 90 degree bend, bubbles go longer distances at the lower part of the pipe and then rises back. It can be also noted that the average void fractions at saturation ratio 0.9 is higher than the average void fractions at saturation ratio 0.75 .

\subsubsection{Buoyancy effect from HSG0 to HSG2}

Figure 13 includes void fraction values for three different positions after the 90 degree bend (HSG0, HSG1 and HSG2) at $1.105 \mathrm{~m} \mathrm{~s}^{-1}$. Bubble volumes show different patterns according to their distance to the bend. At HSG0, peak void fractions are observed at the lower part of pipe section with the effect of 90 degree bend whereas at HSG1 and HSG2, void fractions peaked at the upper parts of the cross section. Moreover, an increase in total void fractions are observed from HSG1 to HSG2 because of buoyancy effect on air bubbles. 

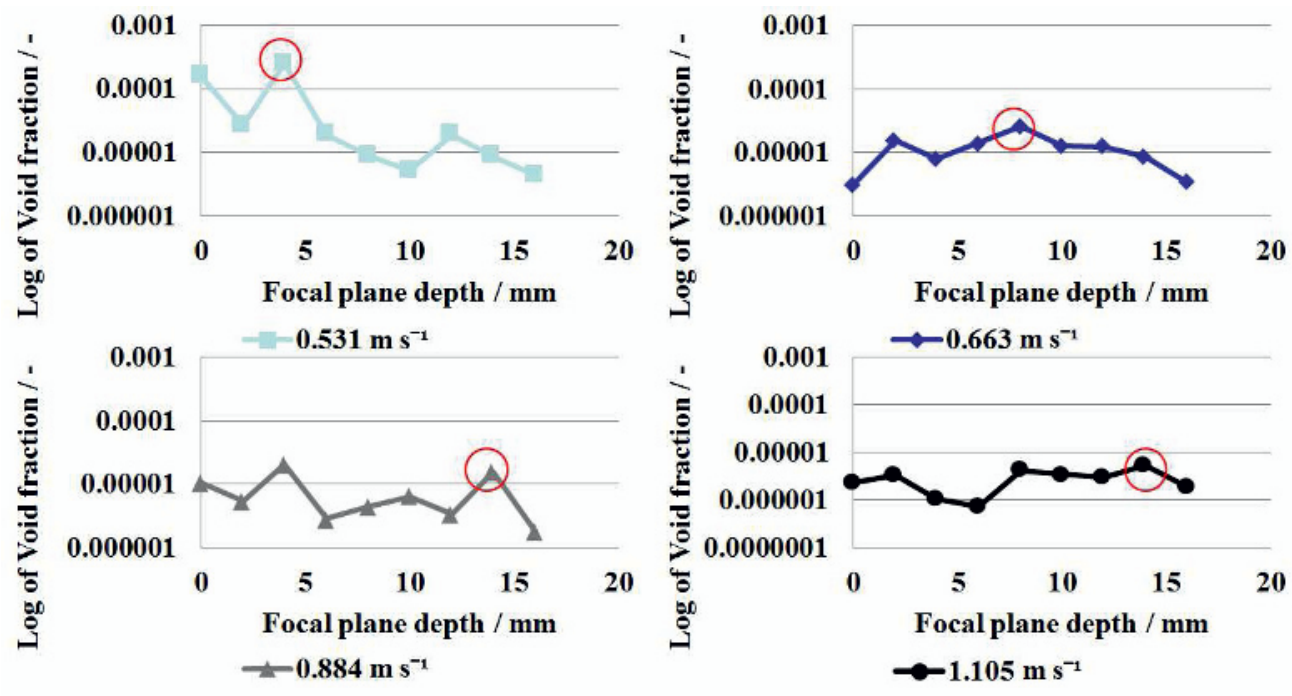

Figure 11. Bend effect at HSG0 at SR0.75
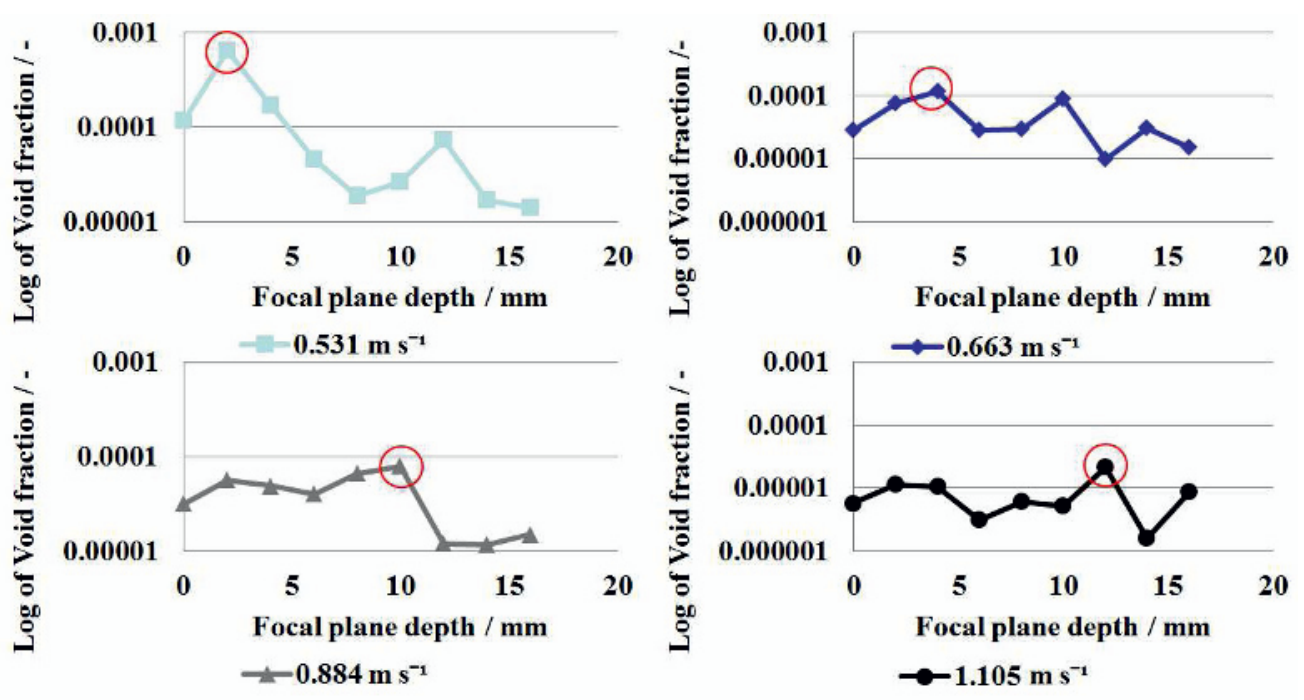

Figure 12. Bend effect at HSG0 at SR0.9

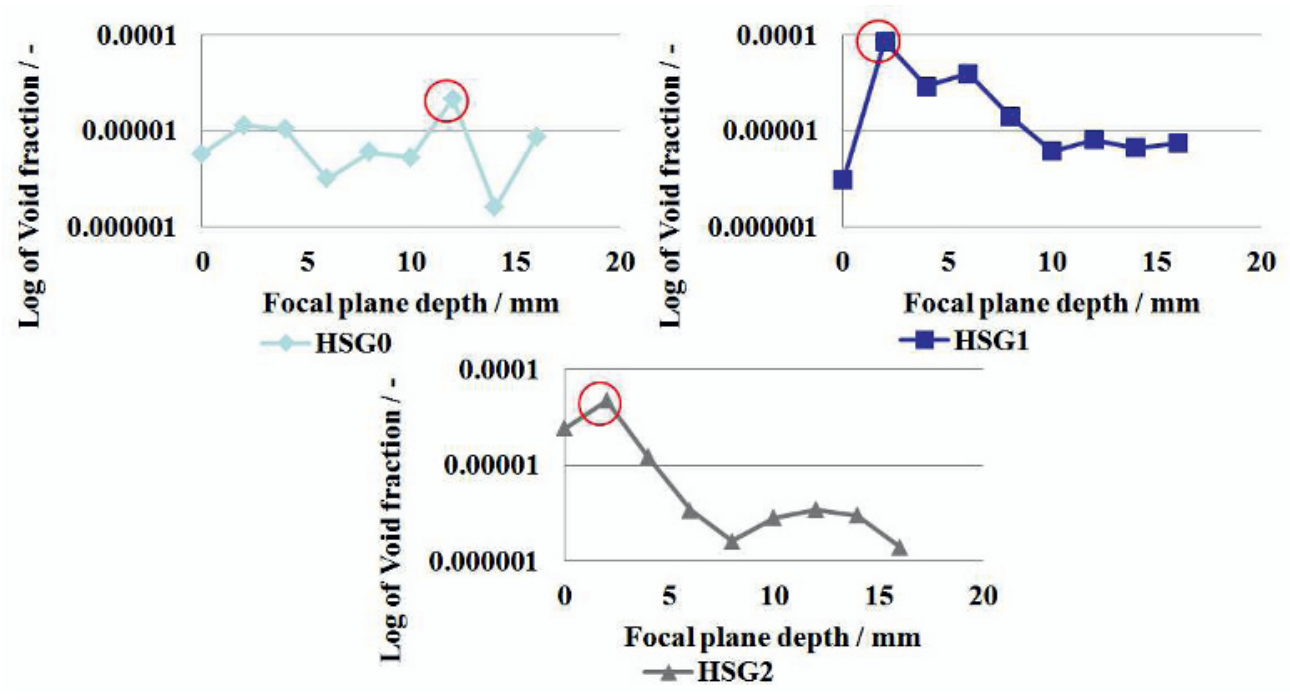

Figure 13. Buoyancy effect along the pipe at $1.105 \mathrm{~m} \mathrm{~s}^{-1}$ 


\section{Average bubble diameters at HSG1}

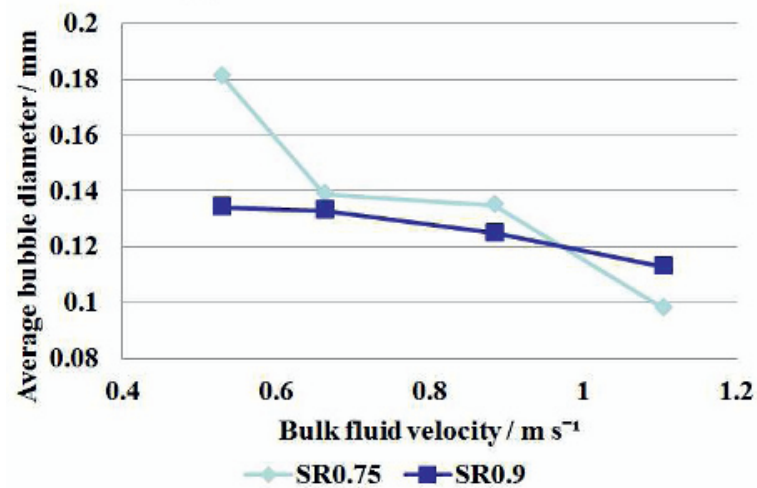

Volumetric void fractions at HSG1

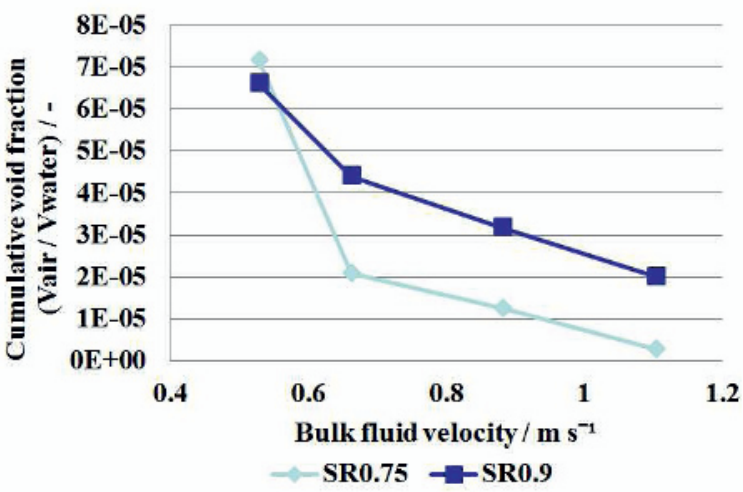

Figure 14. Effect of velocity on average bubble diameter and cumulative void fraction

Average bubble diameter ratios (HSG2/HSG1) at SR0.75

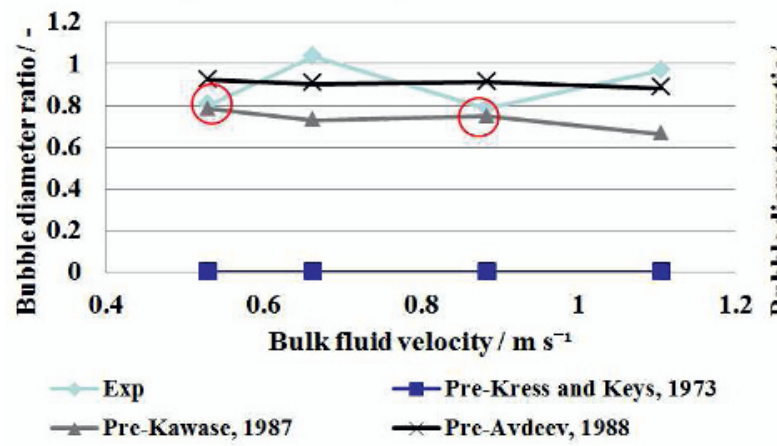

Average bubble diameter ratios (HSG2/HSG1) at SR0.9

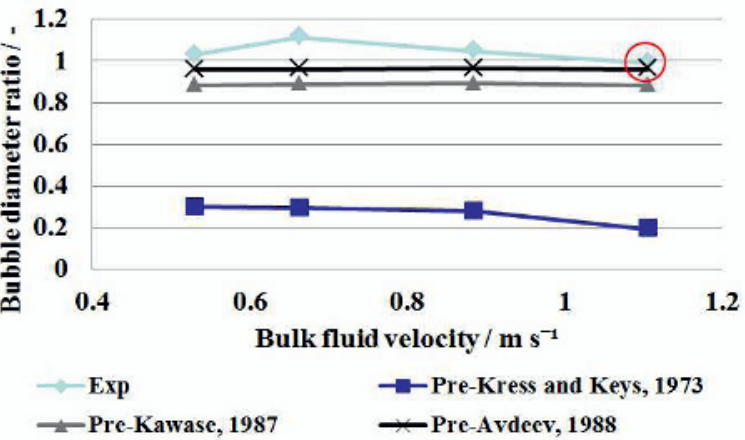

Figure 15. Comparison of dissolution models and experimental data

\subsection{Effect of bulk fluid velocity to average bubble diameter and volumetric void fractions}

Effect of bulk fluid velocity on the average bubble diameter and void fractions are clearly demonstrated in figure 14. The reduction in average bubble diameter and void fractions are observed with increase in bulk fluid velocity.

\subsection{Comparison of dissolution model and experimental data}

Measured and calculated bubble diameter ratios are given in the figure 15 as diameter ratios versus fluid velocity. Bubble size reduction was expected due to concentration differences between phases. However, particular decrease in bubble sizes through horizontal pipe could not be observed from the analysis results. At few flow rates and saturation ratios, reduction in average bubble sizes were observed; on the contrary, general trend shows that average bubble diameter is increasing along the horizontal pipe. Moreover, no consistency or a regular pattern is noticed to verify any conclusion on bubble size changes.

\section{Conclusions}

To conclude, significant data on the effect of flow rate and 90 bend geometry on bubble distribution is obtained. In the first sight glass (HSG0), peak void fraction is getting lower with increase in fluid velocity and void fractions are getting higher at the upper part of the pipe when moved along the horizontal pipe. We can draw a conclusion that there is enough distance to notice strong buoyancy effect in HSG0 at low velocities $\left(0.531 \mathrm{~m} \mathrm{~s}^{-1}\right)$. Moreover, the reduction in average bubble diameter and void fractions are observed with increase in bulk fluid velocity.

The expected bubble diameter reduction which was led by concentration differences between phases could not be detected from the experiment results. No particular patterns on bubble size changes have been observed through sight glasses. Measurements were done with one camera and one sight glass and during the switch of the sight glasses and camera, system fluid was changed. This can cause uncertainty. Ideal experiments would be with two cameras measuring at the same time. The reasons behind this uncertainty should be investigated further. Future experiments will focus on vertical pipeline as well as improving the results of current study. 


\section{Nomenclature}

\section{Abbreviations}

DOF depth of field, mm

SR saturation ratio

TGM total gas measurements

\section{List of symbols}

C

$c_{\mathrm{p}}$

$\mathrm{D}_{\mathrm{AB}}$

d

$k$

$\mathrm{L}$

$\dot{m}$

$P$

$p$

$\mathrm{Q}$

$\mathrm{q}$

$R e=\mathrm{UL} / \mathrm{v}$

$S c=v / D$

$S h=k d_{\mathrm{b}} / D_{A B}$

$\Delta T$

$U$

$X^{\mathrm{T}}$

V

V

v

$\alpha$

gas concentration, $\mathrm{kg} \mathrm{m}^{-3}$

specific heat capasity, $\mathrm{J} \mathrm{Kg}^{-1} \mathrm{~K}^{-1}$

mass diffusivity, $\mathrm{m}^{2} \mathrm{~kg}^{-1}$

diameter, $\mathrm{m}$

coefficient of mass transfer, $\mathrm{m} \mathrm{s}^{-1}$

pipe diameter, $\mathrm{m}$

mass flow rate, $\mathrm{kg} \mathrm{s}^{-1}$

pressure, $\mathrm{Pa}$

partial pressure of gases, $\mathrm{Pa}$

heating load, $\mathrm{kW}$

mass flux, $\mathrm{kg} \mathrm{m}^{-2} \mathrm{~s}^{-1}$

Reynold number, dimensionless

Scmidt number, dimensionless

Sherwood number

temperature diference, ${ }^{\circ} \mathrm{C}$

bulk fluid velocity, $\mathrm{m} \mathrm{s}^{-1}$

temperature dependent henry's constant, $\mathrm{Pa} \mathrm{m}^{-3} \mathrm{~kg}^{-1}$

volume, $\mathrm{mm}^{3}$

kinematic viscosity of liquid, $\mathrm{m}^{2} \mathrm{~s}^{-1}$

saturation ratio, dimensionless

\section{Subscripts}

b

bubble

\section{Superscripts}

$\begin{array}{ll}\text { act } & \text { actual } \\ \text { sat } & \text { saturated } \\ \text { Tbub } & \text { total bubble } \\ \text { Tsam } & \text { total sample } \\ \text { vap } & \text { vapour }\end{array}$

\section{Acknowledgements}

The authors would like to thank the Engineering and Physical Science Council and Spirotech Company for supporting this research.

\section{References}

1. A. M. Fsadni, Y.T. Ge, A.G. Lamers, Applied Thermal Engineering, 31, 2808-2818 (2011).

2. A. M. Fsadni, Y.T. Ge, A.G. Lamers, 45-46, 24-32 (2012).

3. A. M. Fsadni, Y.T., EPJ Web of Conferences, 25, 01016-1-16 (2012).

4. S. Lezhnin, D. Eskin, Y. Leonenko, O. Vinogradov, 39, 483-487 (2003).

5. T. A. Shedd, J. Microlith., Microfab. and Microsyst., 4, 3, 033004-1-8 (2005).

6. W. Gerrard, Solubility of gases and liquids, (New York, Plenium Press 1976).

7. S. Lubetkin, M. Blackwell M, Journal of Colloid and Interface Science, 26, 610-615 (1988).

8. K. T. Sherwood, Mass transfer, McGraw-Hill, (1976).

9. R. H. Perry, D. W. Green, J. O. Maloney, Perry's chemical engineers' handbook, (New York, 1984)

10. Image-Pro Plus, Start-up guide, (Mediacybernetics, 2010) 